\title{
Characteristics, diagnosis, and treatment modality of pediatric patients with cystic echinococcosis: a single centre experience
}

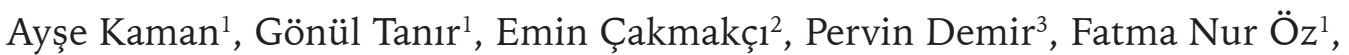 \\ Türkan Aydın Teke ${ }^{1}$, Özge Metin ${ }^{1}$, Zeynep Gökçe Gayretli Aydın ${ }^{1}$, Ayşe Karaman ${ }^{4}$ \\ Departments of ${ }^{1}$ Pediatric Infectious Diseases, ${ }^{2}$ Radiology and ${ }^{4}$ Pediatric Surgery, Dr. Sami Ulus Maternity and Children's \\ Training and Research Hospital; ${ }^{3}$ Department of Biostatistics, Yıldırım Beyazıt University Faculty of Medicine, Ankara, \\ Turkey.E-mail: ayse092003@yahoo.com \\ Received: 26th March 2018, Revised: 25th May 2018, 4th June 2018, 14th December 2018,
} 18th January 2019, Accepted: 7th May 2019

SUMMARY: Kaman A, Tanır G, Çakmakçı E, Demir P, Öz FN, Aydın Teke T, Metin Ö, Gayretli Aydın ZG, Karaman A. Characteristics, diagnosis, and treatment modality of pediatric patients with cystic echinococcosis: a single centre experience. Turk J Pediatr 2019; 61: 704-713.

Cystic echinococcosis (CE) is among the most common zoonotic infections worldwide. Studies about CE are limited in childhood. The aim of this study was to evaluate clinical, radiological and laboratory characteristics of childhood CE at a tertiary care pediatric hospital. Medical records of children with CE were analyzed between January 2005 and January 2015.

A total of 130 patients with a median age of 10.4 years $(I Q R=7.2$-years-13.2 years) were evaluated. The anatomic locations of cysts were as follows; liver (76.9\%), lung $(36.9 \%)$, spleen $(6.2 \%)$, pelvic region $(3.8 \%)$ and kidney $(2.3 \%)$. The most common symptoms were abdominal pain and cough in the patients with liver cysts and lung cysts, respectively. The indirect hemagglutination (IHA) test positivity was $58 \%$. Elevated serum total immunoglobulin E levels were detected in $59 \%$ of the patients. Fourty-four patients with liver CE, 33 patients with lung CE were treated surgically and 23 patients with liver CE were treated with percutaneous aspiration, injection and re-aspiration (PAIR) along with medical treatment. The recurrence was observed in five patients with liver CE.

It was demonstrated that CE mainly involves liver but lung cysts are more frequently symptomatic and prone to be complicated than liver cysts in children. IHA test positivity together with abdominal ultrasonography are useful to diagnose liver CE but thorax CT is usually needed to diagnose lung CE. Liver cysts that are sized greater than $5 \mathrm{~cm}$ are more frequently treated with PAIR or surgery but smaller liver cysts can be treated medically.

Key words: cystic echinococcosis, children, diagnosis, PAIR, treatment.

Cystic echinococcosis (CE) caused by the larval stage (metacestode) of the genus Echinococcus species is among the most common zoonotic infections worldwide. ${ }^{1}$ Humans can acquire infection as intermediate hosts by accidental ingestion of contaminated water or uncooked food, or direct hand-to-mouth fecal transmission (especially common in children) of the infective eggs. Because only $10 \%-20 \%$ of CEs are diagnosed in children younger than 16 years of age, studies concerning CE are limited in childhood. ${ }^{2}$

The aim of the study was to assess demographical, clinical features and radiological characteristics including anatomical location, size, number, stage of cysts, diagnostic and therapeutic modalities, 
complications and outcome of pediatric patients in the setting of a tertiary care pediatric hospital.

\section{Material and Methods}

Patients with CE were evaluated retrospectively between January 2005 and January 2015 in a pediatric hospital in Ankara, Turkey. Dr. Sami Ulus Children's Hospital is a tertiary-care training and research hospital with 416 beds and acts as a referral pediatric center for the entire country. The study protocol was approved by the Institutional Ethics Committee with the decision number of 2018/1604 and written consents of parents were obtained. Data regarding age, sex, socio-demographic details, clinical presentations, anatomical location, size, number of cysts determined by imaging modalities, laboratory evaluations, duration of treatment, complications and outcome were recorded. The diagnosis of CE was made based on the suggestive radiologic findings and/ or positive serological tests. Laboratory tests including indirect hemagglutination (IHA), total immunoglobulin E (IgE) level, total eosinophil count, aspartate aminotransferase (AST), alanine aminotransferase (ALT) were eveluated. IgE was determined using $\mathrm{N}$ Latex IgE mono (N IgE) BN II/BN ${ }^{\circledR}$ ProSpec System (Germany). The upper limit of serum IgE concentration was considered $200 \mathrm{IU} / \mathrm{ml}$. Fumouse Laboratories Kit ${ }^{\circledR}$, (France) was used for IHA. Because IHA test was not available before 2011, we couldn't use this test in all patients. At least one of the following imaging studies; posterior-anterior chest radiography, thorax and/or abdominal ultrasonography (US), thorax and/or abdominal computed tomography (CT) and abdominal magnetic resonance imaging (MRI) were performed on the patients. US images of cysts were classified according to both Gharbi et al. classification and World Health Organization Informal Working Group on Echinococcosis (WHOIWGE) classification by the same experienced pediatric radiologist. ${ }^{3}$

Patients were treated as medically and/or interventionally and/or surgically. Albendazole was given at a dose of $15 \mathrm{mg} / \mathrm{kg} /$ day, orally for at least 3 months, separated by intervals of one week. Liver enzyme levels and complete blood counts were monitored for side effects in all patients. As interventional treatment, percutanous aspiration was performed with 18-20-gauge needle. First, fluid in cyst was aspirated and then hypertonic saline or sterile alcohol (95\% concentration) was injected into cyst cavity and reaspiration (PAIR) were applied.

Total or partial pericystectomy and capitonnage had been performed as surgical treatment. The terminology of 'radical surgery' is used for lobectomy and splenectomy. Resected specimens were examined by the pathology department.

Data were entered to a database and statistical analyses were performed using IBM SPSS Statistics 21.0 (IBM Corp. Released 2012. IBM SPSS Statistics for Windows, Version 21.0. Armonk, NY: IBM Corp.) and MS-Excel 2007 programmes. The variables for instance age and duration of treatment were investigated using Shapiro-Wilk's test to determine whether or not they were normally distributed. Descriptive analyses were presented using means $\pm S D$, and as medians [interquartile range (IQR) was the 1st and 3rd quartiles] for the non-normally distributed variables. Frequencies were given to summarize the categorical variables. The Mann-Whitney $U$ test was used to compare median of age values according to sex. The appropriate one of Pearson Chi-square, Fisher's exact and Continuity Correction test results were given to evaluate differences between the categorical two variables. The Kruskal Wallis $\mathrm{H}$ test was used to evaluate whether there were any differences between the involved organ with respect to the age, IHA and eosinophil values. The bonferroni adjustment was used for pairwise comparisons. Cyst stage and IHA were compared with Fisher exact test Kruskal Wallis nonparametric analyses. A p-value less than 0.05 was considered as statistically significant result.

\section{Results}

A total of 130 patients diagnosed with CE were evaluated in this study. Number of boys and girls were $68(52.3 \%)$ and $62(47.7 \%)$, respectively. Age distribution was from 2.6 years to 17.5 years. Mean age and median age 
were 10.3 years (SD 3.5 years old) and 10.4 years (IQR: 7.2 years - 13.2 years), respectively. Distribution of age according to gender was similar $(p=0.255)$. Eighty-six $(66.2 \%)$ patients were from urban and $44(33.8 \%)$ patients were from rural areas. Sixty-three $(48.5 \%)$ patients had a history of intimate contact with animals. Family history of known CE was observed in only six patients.

The anatomic locations of the cysts were as follows: liver in $100(76.9 \%)$ patients, lungs in $48(36.9 \%)$ patients, both liver and lung in $21(16.1 \%)$ patients, spleen in $8(6.2 \%)$ patients, pelvic region in $5(3.8 \%)$ patients, and kidney in $3(2.3 \%)$ patients. One patient with disseminated $\mathrm{CE}$ had multiple lesions in bilateral lungs, liver, spleen, kidney, pelvic cavity and sternocleidomastoid muscle. The pertcentage of liver cysts in the right lobe, left lobe and both lobes were $57 \%, 21 \%$ and $22 \%$, respectively. The pertcentage of lung cysts in the right lung, left lung and both lungs were $58 \%, 25 \%$ and $17 \%$, respectively. The age distribution of the patients with liver, lung and both lung and liver cysts was not different $(\mathrm{p}=$
0.48). Boy to girl ratios with CE involving liver, lung and both liver and lung were 0.8, 0.7 and 4.6 , respectively. Boys had significantly higher likelihood of having both liver and lung lesions than having only liver or only lung lesions $(p=0.023$ and $p=0.028)$. Multiple cysts were identified in $30(39 \%)$ patients with liver $\mathrm{CE}$, and in 3 (12.5\%) patients with lung CE. Multiple cysts in the liver were more frequent than in the lung $(p=0.03)$. Cyst sizes were smaller than $5 \mathrm{~cm}$ in 47 patients, and greater than $10 \mathrm{~cm}$ in 31 patients. The remaining 52 patients had cysts sized between 5 to $10 \mathrm{~cm}$.

Thirty-four (26\%) patients had no symptoms related to $\mathrm{CE}$ and they were diagnosed incidentally. Referral symptoms of the patiens are demonstrated on Table I. By presence of symptoms, there was a statistically significant difference between lung $\mathrm{CE}$ and liver CE $(p=0.007)$, but there were no statistically significant differences between lung $\mathrm{CE}$ and lung+liver $\mathrm{CE}$ and between liver $C E$ and lung+liver CE $(p=0.455$ and $\mathrm{p}=0.412$, respectively). There was no significant difference between symptomatic

Table I. Presenting Symptoms of Patients with Liver Cystic Echinococcosis (CE), Lung CE and Liver+ Lung CE.

\begin{tabular}{lccc}
\hline & $\begin{array}{c}\text { Liver }(\mathrm{n}=77) \\
\mathrm{n}(\%)\end{array}$ & $\begin{array}{c}\text { Lung }(\mathrm{n}=24) \\
\mathrm{n}(\%)\end{array}$ & $\begin{array}{c}\text { Liver+ Lung }(\mathrm{n}=17) \\
\mathrm{n}(\%)\end{array}$ \\
\hline Abdominal pain & $37(75.5)$ & $4(17.4)$ & $4(28.6)$ \\
Cough & $2(4.1)$ & $15(65.2)$ & $9(64.3)$ \\
Fever & $4(8.2)$ & $11(47.8)$ & $4(28.6)$ \\
Chest Pain & - & $9(39.1)$ & $4(28.6)$ \\
Pneumonia symptoms & - & $7(30.4)$ & $5(35.7)$ \\
Abdominal mass/distension & $8(16.3)$ & - & - \\
Jaundice & $1(2.0)$ & - & - \\
Weight loss & $3(6.1)$ & $3(13.0)$ & - \\
Nausea and vomiting & $2(4.1)$ & - & - \\
Hemoptysis & - & $2(8.7)$ & - \\
Weakness & $3(6.1)$ & - & $1(7.1)$ \\
Dyspnea & - & $2(8.7)$ & $1(7.1)$ \\
Expectoration & - & $1(4.3)$ & $1(7.1)$ \\
Anorexia & $1(2.0)$ & - & - \\
Itching & $1(2.0)$ & - & $1(7.1)$ \\
Hydatid vomica & - & - & $14(82.4)$ \\
\hline Total & $49(63.6)$ & $23(95.8)$ & \\
\hline & & & - \\
\hline
\end{tabular}


and asymptomatic patients according to cyst sizes or cyst stages $(p=0.35$ and $p=0.98$, respectively).

Antibody test to E. granulosus was performed in $86 \%(112 / 130)$ of the patients. Positive and negative results were detected in $58 \%$ $(65 / 112)$ and $42 \%(47 / 112)$ of the patients, respectively. IHA results are demonstrated in Table II and Table III. Total IgE level was performed in 74 of 130 (56\%) patients and detected as high in 44 (59\%) of the patients. Serum IHA positivity was found significantly high in patients whose total IgE levels were elevated $(p=0.004)$. Absolute eosinophil count was greater than $500 / \mathrm{mm}^{3}$ in 45 (34.7\%) patients and greater than $1500 / \mathrm{mm}^{3}$ in $35(26 \%)$ patients. The absolute eosinophil count was not different between symptomatic and asymptomatic patients $(\mathrm{p}=0.335)$ and was similar among the patients who had liver, lung and liver+lung CE $\left(X^{2}=5.351 ; \mathrm{p}=0.069\right)$.
The median and mean eosinophil counts and liver function test values are shown in Table IV. Acccording to the US image, CE stages of liver cysts and lung cysts are shown on Figure 1 and Figure 2, respectively. In addition to abdominal US, 40 (40\%) patients with liver CE had abdominal CT, 8 (8\%) patients had abdominal MRI. Thorax CT was performed on $43(89 \%)$ patients with lung CE.

Thirty two (24\%) patients had a complication on admission. Number of complications in patients were as follows, 22 (16\%) cyst rupture, $8(6 \%)$ secondary bacterial infection and $2(1 \%)$ cyst rupture plus secondary bacterial infection. Complications occured in $6(7.8 \%)$ patients with liver CE, 14 (58.3\%) patients with lung $\mathrm{CE}$ and 7 (41.2\%) with both liver and lung CE. Complications were statistically higher in patients with lung CE than the patients with liver CE $(\mathrm{p}<0.001)$.

Table II. Indirect Hemagglutination Test Results Comparison Among the Patients with Liver, Lung and Liver+ Lung Cystic Echinococcosis.

\begin{tabular}{lccccc}
\hline & $\begin{array}{c}\text { Liver } \\
\mathrm{n}(\%)\end{array}$ & $\begin{array}{c}\text { Lung } \\
\mathrm{n}(\%)\end{array}$ & $\begin{array}{c}\text { Liver +Lung } \\
\mathrm{n}(\%)\end{array}$ & \multirow{2}{*}{$X^{2}$} & $\mathrm{p}$ \\
\hline Negative & $30(45.5)^{\mathrm{a}}$ & $12(54.5)^{\mathrm{b}}$ & $1(7.1)^{\mathrm{a}, \mathrm{b}}$ & 8.718 & 0.013 \\
Positive & $36(54.5)$ & $10(45.5)$ & $13(92.9)$ & & \\
\hline
\end{tabular}

a,b: $\mathrm{p}<0.05$, define the differences

Table III. Indirect Hemagglutination (IHA) Positivity or Negativity According to Activity of Hydatic Cysts in Liver and Lung.

\begin{tabular}{|c|c|c|c|c|c|}
\hline \multirow[b]{2}{*}{ Involved organ } & \multirow[b]{2}{*}{ Disease activity } & \multicolumn{2}{|c|}{ IHA Titers } & \multirow[b]{2}{*}{$X^{2}$} & \multirow[b]{2}{*}{$\mathrm{p}$} \\
\hline & & $\begin{array}{c}\text { Negative } \\
\text { n (\%) }\end{array}$ & $\begin{array}{c}\text { Positive } \\
\text { n (\%) }\end{array}$ & & \\
\hline \multirow{2}{*}{ Liver $(n=85)$} & Active & $27(39.7)$ & $41(60.3)$ & \multirow{2}{*}{0.003} & \multirow{2}{*}{0.956} \\
\hline & Inactive & $6(35.3)$ & $11(64.7)$ & & \\
\hline \multirow{2}{*}{ Lung $(n=40)$} & Active & $15(39.5)$ & $23(60.5)$ & \multirow[b]{2}{*}{ - } & \multirow{2}{*}{0.519} \\
\hline & Inactive & $0(0.0)$ & $2(100.0)$ & & \\
\hline
\end{tabular}

Table IV. Mean and Median Eosinophil Count, Immunoglobulin (Ig) E, Aspartate Aminotransferase (AST), Alanine Aminotransferase (ALT) Values of Patients with Cystic Echinococcosis (CE).

\begin{tabular}{lccc}
\hline & Number of patients & Median $(\mathrm{IQR})$ & Mean \pm SD \\
\hline Eosinophil count $\left(/ \mathrm{mm}^{3}\right)$ & 130 & $280.0(575.0)$ & $641.3 \pm 1320.7$ \\
$\lg$ E $(\mathrm{IU} / \mathrm{ml})$ & 74 & $291.0(860.0)$ & $1855.8 \pm 6266.0$ \\
AST $(\mathrm{IU} / \mathrm{l})$ & 130 & $25.0(12.0)$ & $29.9 \pm 21.9$ \\
ALT $(\mathrm{IU} / \mathrm{l})$ & 130 & $17.0(11.0)$ & $22.0 \pm 18.4$ \\
\hline
\end{tabular}




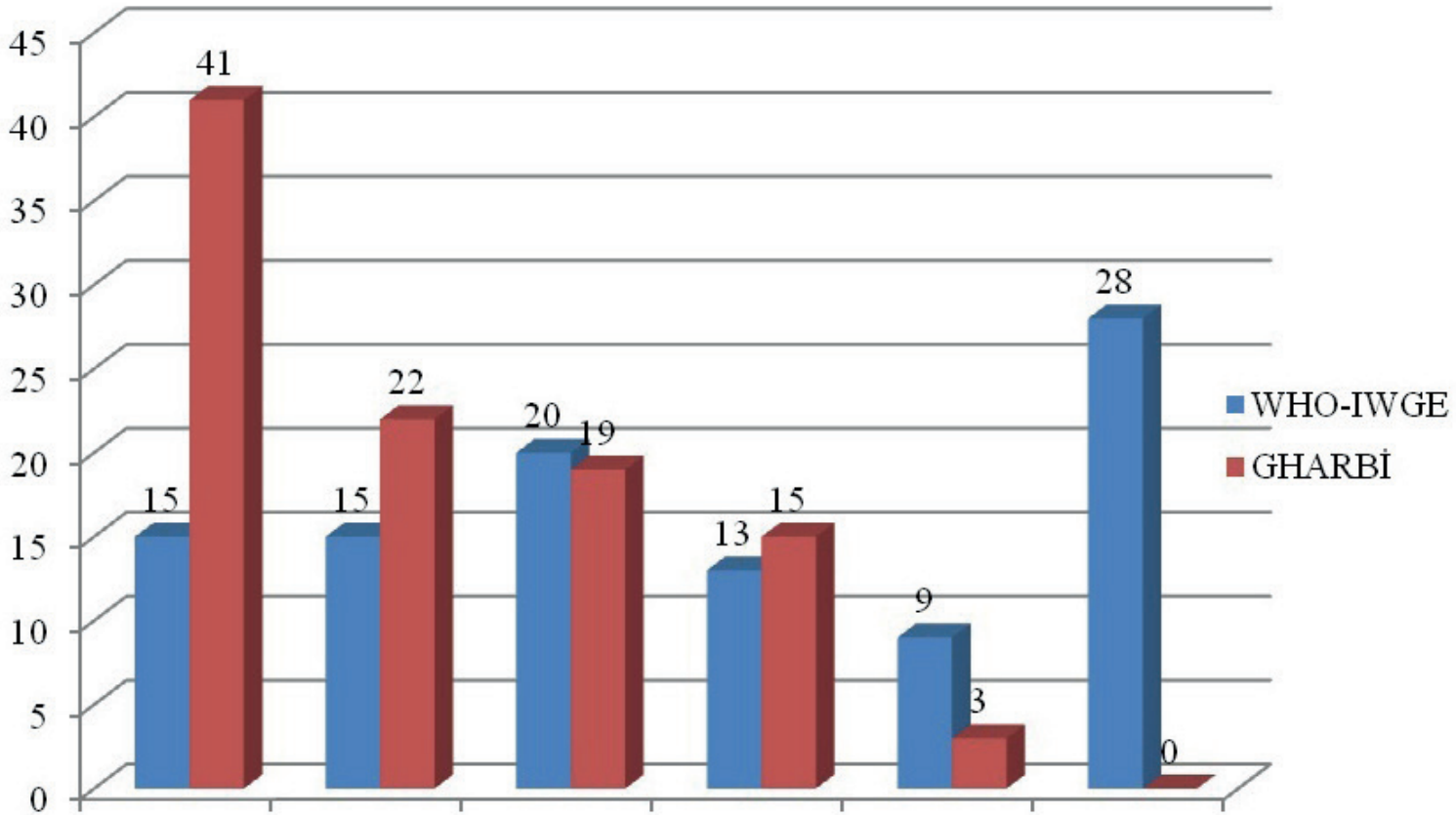

CE1-Type 1 CE2-Type 2 CE3-Type 3 CE4-Type 4 CE5-Type 5

CL

Fig. 1. Number of Liver Cysts acording to Gharbi and WHO-IWGE Classification.

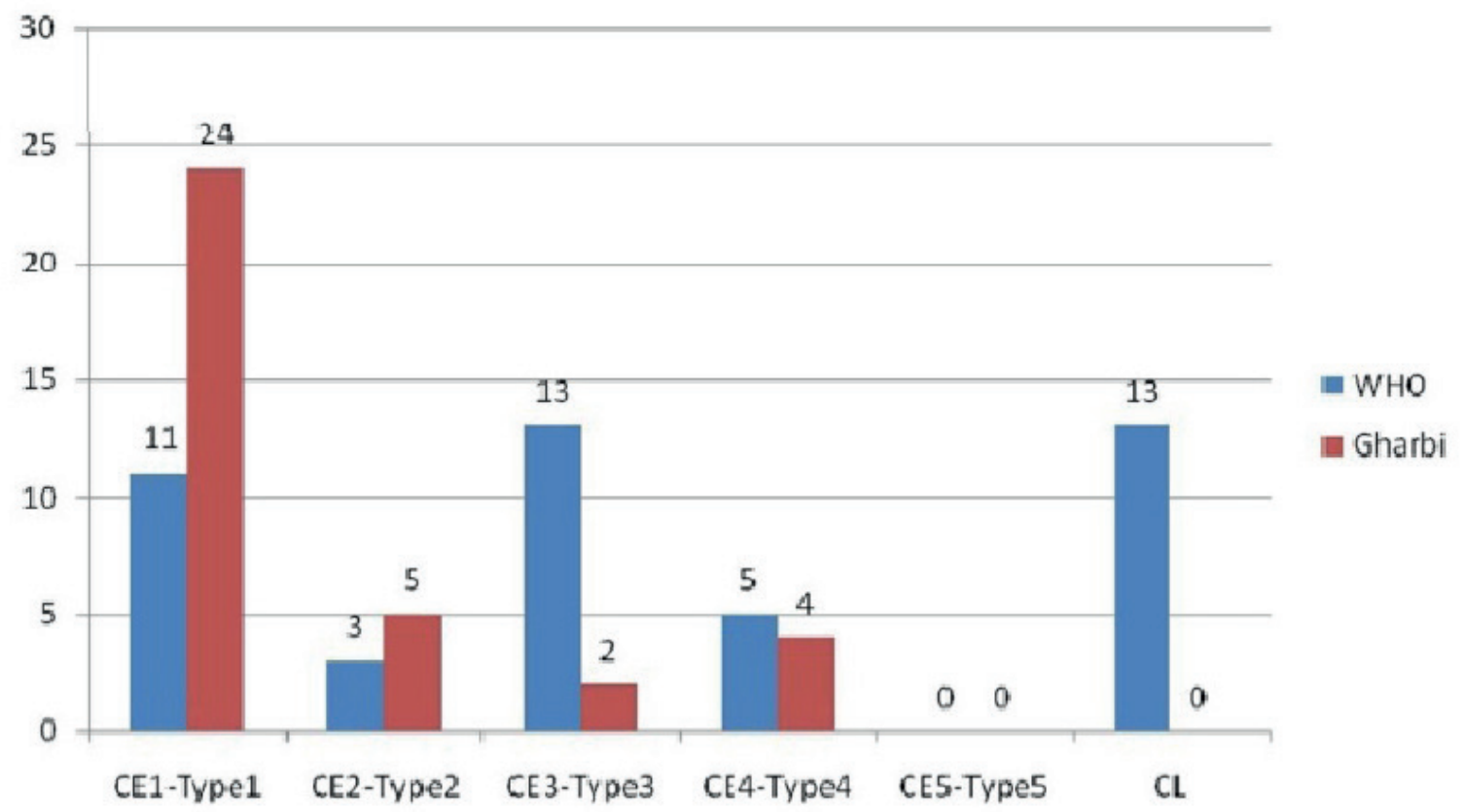

Fig. 2. Number of Lung Cysts numbers acording to Gharbi and WHO-IWGE Classification.

Ninety-eight $(75.4 \%)$ patients were admitted to hospital, $32(24.6 \%)$ patients were treated as outpatients. Mean duration of drug therapy with albendazole for all of the patients were $3.85 \pm 1.74$ months (range 1-12 months). Medical treatment alone was used in 30 (30\%) patients with liver CE (mean duration of therapy was $4.23 \pm 2.07$ months, range 1-9 months) and in 15 (31\%) patients with lung CE (mean duration of therapy was 
$3.73 \pm 1.62$, range: $1-7$ months). Fourty-four (44\%) patients with liver CE and 33 (68\%) patients with lung CE were treated surgically. PAIR was performed in 23 (23\%) patients with liver CE. The remaining three patients with liver CE were followed-up without any treatment. Treatment modalities according to the number and size of cysts in liver and lung CE are demonstrated in Table V. Treatment modalities according to the WHO-IWGE stages of cysts are shown in VI. Cystectomy and capitonage were performed on all patients with liver CE and almost all patients with lung CE except for one patient who had lobectomy. Two patients with spleen CE recieved a splenectomy. A total of 22 resected specimens were examined by the pathology department (13 liver, 6 lung, 2 spleen, 1 kidney) and all of the histopathological examination of these specimens revealed a fibrotic cyst wall containing chronic inflammation. Daughter vesicles and germinative membranes were observed in six of the examined specimens. Recurrence of cyst was detected in three surgically treated patients with liver CE (one of them was reoperated) and two patients with liver CE treated with PAIR (one of them had surgery). Three patients with recurrent disease were treated medically with success. Albendazole was well tolerated, except for seven out of 112 patients whose transaminase levels were monitored during medical treatment $(7 / 122=6.2 \%)$. Treatment of seven patients were interrupted due to liver enzyme levels elevation. Except for ten patients all others came to outpatient visits at least once. Median time of follow-up was 12 months (range: 1-98 months).

\section{Discussion}

$\mathrm{CE}$ is a public health problem in endemic areas, especially in countries of the temperate zones (South America, China, Mediterranean, the Middle East, Australia and some Balkan countries). ${ }^{4}$ Exposure of humans to $\mathrm{CE}$ is common in Turkey, because the majority of people live in rural areas and are engaged in animal husbandry. ${ }^{5}$ In a seroepidemiological study conducted between 2000-2002 in a rural area of Turkey, 2242 resident subjects, aged from 10 to 90 years, were screened by indirect fluorescence antibody (IFA) and enzymelinked immunosorbent assay (ELISA), and the seropositivity rate was $2.7 \% .^{6}$ In an another epidemiological study from Turkey, 1133 adults were included and 78 (6.9\%) of the patients were seropositive. ${ }^{7}$ According to Ministry of Health records, the estimated incidence rate of $\mathrm{CE}$ is $0.8-2.0 / 100.000$ between 1990 to 2005

Table V. Treatment Choice in Relation to Number and Sizes of Cyst.

\begin{tabular}{|c|c|c|c|c|c|c|c|c|c|}
\hline & \multicolumn{2}{|c|}{ Cyst number } & \multirow[b]{2}{*}{$X^{2}$} & \multirow[b]{2}{*}{$\mathrm{p}$} & \multicolumn{3}{|c|}{ Cyst size } & \multirow[b]{2}{*}{$X^{2}$} & \multirow[b]{2}{*}{$\mathrm{p}$} \\
\hline & $\begin{array}{c}\text { One cyst } \\
\mathrm{n}(\%)\end{array}$ & $\begin{array}{c}\geq 2 \text { cyst } \\
\mathrm{n}(\%)\end{array}$ & & & $\begin{array}{l}<5 \mathrm{~cm} \\
\mathrm{n}(\%)\end{array}$ & $\begin{array}{l}5-10 \mathrm{~cm} \\
\mathrm{n}(\%)\end{array}$ & $\begin{array}{c}>10 \mathrm{~cm} \\
\mathrm{n}(\%)\end{array}$ & & \\
\hline \multicolumn{10}{|l|}{ Liver } \\
\hline Medical treatment & $15(27.3)$ & $15(35.7)$ & & & $21(56.8)^{a}$ & $7(17.1) b$ & $2\left(10.5^{b}\right.$ & & \\
\hline $\begin{array}{l}\text { Surgery }+ \text { medical } \\
\text { treatment }\end{array}$ & $27(49.1)$ & $17(40.5)$ & & & $6(16.2)^{\mathrm{a}}$ & $22(53.7)^{b}$ & $16(84.2)^{\mathrm{b}}$ & & \\
\hline $\begin{array}{l}\text { Percutaneous } \\
\text { aspiration, injection } \\
\text { and re-aspiration } \\
\text { (PAIR) + medical } \\
\text { treatment }\end{array}$ & $13(23.6)$ & $10(23.8)$ & 0.939 & 0.625 & $10(27.0)^{\mathrm{a}}$ & $12(29.3)^{\mathrm{a}}$ & $1(5.3)^{\mathrm{a}}$ & 30.388 & $<0.001$ \\
\hline \multicolumn{10}{|l|}{ Lung } \\
\hline Medical treatment & $12(31.6)$ & $3(30.0)$ & & & $8(38.1)$ & $5(29.4)$ & $2(20.0)$ & & \\
\hline $\begin{array}{l}\text { Surgery }+ \text { medical } \\
\text { treatment }\end{array}$ & $26(68.4)$ & $7(70.0)$ & 3.891 & 0.143 & $13(61.9)$ & $12(70.6)$ & $8(80.0)$ & 2.616 & 0.624 \\
\hline
\end{tabular}

$\mathrm{a}, \mathrm{b}$ : define the differences

$\mathrm{p}<0.001$ is the result for Pearson Chi-square test of the $3 \times 3$ table for liver cysts treatment and cyst size 
Table VI. The Choice of Treatment According to the World Health Organization Informal Working Group on Echinococcosis (WHO-IWGE) Stages.

\begin{tabular}{|c|c|c|c|c|c|c|c|}
\hline Treatment & n $(\%)$ & $\begin{array}{c}\text { CE } 1 \\
\text { n (\%) }\end{array}$ & $\begin{array}{c}\text { CE } 2 \\
\text { n (\%) }\end{array}$ & $\begin{array}{l}\text { CE } 3 \\
\text { n (\%) }\end{array}$ & $\begin{array}{c}\text { CE } 4 \\
\text { n }(\%)\end{array}$ & $\begin{array}{l}\text { CE } 5 \\
\text { n (\%) }\end{array}$ & $\mathrm{CL}$ \\
\hline \multicolumn{8}{|l|}{ Liver $(n=100)$} \\
\hline Medical treatment & $30(30.9)$ & $6(40.0)$ & $5(33.3)$ & $7(35.0)$ & $4(30.8)$ & $4(44.4)$ & $4(14.3)$ \\
\hline Surgery + medical treatment & $44(45.4)$ & $6(40.0)$ & $8(53.3)$ & $7(35.0)$ & $7(53.8)$ & $1(11.1)$ & $15(53.6)$ \\
\hline $\begin{array}{l}\text { Percutaneous aspiration, } \\
\text { injection and re-aspiration } \\
+ \text { medical treatment }\end{array}$ & $23(23.7)$ & $3(20.0)$ & $2(13.4)$ & $6(30.0)$ & $2(15.4)$ & $1(11.1)$ & $9(32.1)$ \\
\hline \multicolumn{8}{|l|}{ Lung $(\mathrm{n}=48)$} \\
\hline Medical treatment & $15(31.3)$ & $5(38.4)$ & $0(0.0)$ & $5(38.4)$ & $1(20.0)$ & - & $4(30.8)$ \\
\hline Surgery + medical treatment & $33(68.7)$ & $8(61.6)$ & $3(100.0)$ & $8(61.6)$ & $4(80.0)$ & - & $10(69.2)$ \\
\hline
\end{tabular}

in Turkey. ${ }^{5}$ Studies of CE in children are rare and the global incidence is unknown. Pediatric prevalence of CE was found as $0.15 \%$ in a study from Turkey. ${ }^{8} \mathrm{CE}$ may be detected at any age however the incidence of disease increases with age. Because of the slow growth pattern of the disease, the infection that was acquired in chilhood might be asymptomatic for many years and clinical signs of CE might appear as the disease progresses with age. ${ }^{1,4,9}$ It was reported that most of the patients with CE in studies including both children and adults, were females. ${ }^{10,11}$ However in some studies including both children and adults and in pediatric studies, male gender dominance was found. ${ }^{4,12}$ In a five years retrospective study from Turkey that included 152 pediatric and adolescent patients with liver and/or lung CE, most of the patients were older than 9 years and male. Authors suggested that older and male children have an increased risk of being infected by E. granulosus during their life. ${ }^{13}$ Unsimilar to these reports, male or female dominance was not detected at any age in this study. Our finding is consistent with the 2012 European Centre for Disease Prevention and Control (ECDC) annual report that showed that there were no major differences in echinococcosis case rates by gender. ${ }^{14}$

The risk factors for $\mathrm{CE}$ in rural areas are; uncontrolled sheepherd dogs who have close contact both livestocks and people, uncontrolled slaughter of livestock, and unsanitary living conditions. ${ }^{15}$ However it has been reported that, stray dogs without veterinary control, shed E. granulosus eggs in playgrounds or gardens of homes and schools thus children may encounter with Echinococcus in urban areas. ${ }^{8,16}$ In our study most of the patients with $\mathrm{CE}$ were living in urban areas. Because our study is not an epidemiological field study, this finding can not be generalized.

Echinococcal cysts can localize in virtually any organ and structure, such as abdominal or pleural cavities, kidney, spleen, bone, brain, eye, ovary, testis, and pancreas. ${ }^{17}$ Liver CE were found more frequently than lung $\mathrm{CE}$ at a ratio of $2: 10^{2,4}$ In our study, most of the patients had liver involvement and the distribution of ages according to the liver or lung CE was similar. The symptoms of CE depend on the affected organ, cyst size and condition of the cysts. Most of the patients with $\mathrm{CE}$ are asymptomatic and cysts are diagnosed incidentally by radiological studies done for unrelated reasons. ${ }^{13,17}$ The most common symptoms of hepatic CE are abdominal pain, hepatic enlargement (with or without palpable mass), jaundice, nausea, and vomiting. Cough, chest pain, fever or allergic skin rashes are among the presenting symptoms of lung cysts.,13 Rupture with resultant secondary bacterial infection, fistula development with adjacent structures (e.g. biliary tract, intestine, and bronchus), anapylaxis or mass effect on adjacent tissues are the major complications of CE. ${ }^{17}$ In the present study, lung cysts were more frequently symptomatic than liver cysts and the most 
common symptoms were cough and abdominal pain, respectively. Furthermore, it was found that, complications were statistically higher in patients with lung cysts than the liver group.

The diagnosis of CE is based on combined use of serologic tests and imaging studies. ${ }^{10,18}$ Among the biochemical tests; elevated serum transaminases, bilirubin levels and gammaglutamyl transferase levels may be observed in patients who have biliary obstruction. When liver cyst rupture in the biliary tract, significant elevation in serum gamma-glutamyl transferase, alkaline phosphatase levels may be detected. Eosinophilia may also be observed in patients who have ruptured cysts. ${ }^{17}$ AST and ALT levels were in normal limits in our series. Mild to moderate eosinophilia and increased IgE level that were unrelated to the presence of sypmtoms or localization of CE were found in our study. We suggested that routine laboratory investigations are usually nonspecific in patients with CE. Several serological methods have been developed for the diagnosis of CE. The currently available antibody detection assays include ELISA, IHA, latex agglutination tests, and immunoblot tests. Many factors including quality of the antigen preparation and host factors, such as the patient's immune status, can influence sensitivity of these tests. Immune responses depend on the location, cyst wall intactness and activity of the cysts. The patients who have cysts that inactive staged and calcified thick walled are often seronegative. ${ }^{10,17,19}$ The IHA positivity of the patients who had both lung and liver cysts was found statistically higher than the patients with only liver or lung CE in our study. However, IHA positivity was not different between the patients who had active or inactive cysts.

Pulmonary CE can be diagnosed with direct radiography. Calcification can be seen on radiographs in up to $30 \%$ of CE. CT, MRI and US are useful for diagnosis of deep-seated cysts in all organs. US, a non-invasive method, has a sensitivity of about $88-98 \%$ in detecting liver and other cysts in the abdominal cavity but it may not detect pulmonary CE accurately. ${ }^{1,10,18}$ Abdominal US is the most widely used imaging technique for echinococcosis because of its widespread availability and usefulness for defining number, site, dimensions, and vitality of cysts. ${ }^{1,10}$ US is not only helpful for diagnosis of $\mathrm{CE}$, it is particularly suitable for guiding to interventional treatment and for follow-up monitoring. ${ }^{19}$ Several classification schemes exist for staging $\mathrm{CE}$, the WHO developed a standardized classification system that was revised by the WHO-IWGE in 2003. ${ }^{3}$ This classification system is intended to be used for both the epidemiological field and in clinical settings to use in stage-specific treatment. It was developed on the basis of liver CE but, in a variety of cases, it could also be applied in the management and follow-up of cysts located otherwhere. .,17 Abdominal US was used as single imaging method to diagnose liver $\mathrm{CE}$ in more than half of the patients in our study. Abdominal CT was performed as an additional modality for the purpose of evaluation of the cyst before surgery or interventional treatment. Abdominal MRI was required in only small number of the patients with complications. In the present study, nearly all of the patients with lung CE were diagnosed with thorax CT.

Surgery, chemotherapy with benzimidazole compounds (albendazole and mebendazole) and PAIR are the treatment modalities of CE. ${ }^{1,20}$ It was reported that medical treatment is indicated for inoperable cases in primary lung $\mathrm{CE}$ and liver $\mathrm{CE}$ with multiple cysts, and for reducing cyst pressure. ${ }^{10}$ PAIR is a minimally invasive, safe, non-surgical drainage technique with low morbidity and mortality rates that can be used for the treatment of CE except for lung CE. ${ }^{1,20,21}$ Reported PAIR indications are inoperable patients, relapse after surgery or failure with medical treatment alone, cysts sized $>5 \mathrm{~cm}$, cysts staged CE 1 and CE3. ${ }^{1,19}$ Surgical removal of CE with partial or total cystectomy, remains the best curative treatment modality. Surgery is the first choice for; large liver cysts (in particular $>10 \mathrm{~cm}$ ) and those staged CE2 and CE3b, cysts that contain multiple daughter vesicles (CE2 and $\mathrm{CE} 3 \mathrm{~b}$ ), cysts destroying the biliary ducts, and cysts that localized superficially and those at risk of spontaneous rupture or infected (when interventional treatment is not available).,21 It was found that, only the size of liver cysts was a determining factor for surgical treatment and smaller cysts might be treated alone with medical treatment in this study. Liver cysts that were sized $>5 \mathrm{~cm}$ were more 
frequently treated with PAIR or surgery in our study. None of the patients who were treated medically alone experienced a recurrence. Recurrence rate of nonsurgically treated cysts was not higher than cysts which were treated surgically in this study. It was reported that approximately a third of patients treated with benzimidazole drugs were cured and 30\%-50\% of them demonstrated significant regression of cyst size and symptoms. ${ }^{1}$ Because of the small number of the patients in each WHO-IWGE stages, we couldn't evaluate the usefullness of these classification systems for the choice of treatment.

In conclusion; in the environment of a tertiary pediatric hospital, CE was seen mostly in older children and the liver was the most common location in all ages. Lung cysts were more frequently symptomatic and prone to be complicated than the liver cysts. IHA positivity was highest in patients who had cysts in more than one location. Abdominal US was a useful imaging modality to diagnose liver $\mathrm{CE}$, while lung CE was mainly diagnosed by thorax CT. Smaller liver cysts could be treated succesfully by medical treatment alone, but liver cysts that were sized $>5 \mathrm{~cm}$ were more frequently treated with PAIR or surgery. Further studies including larger number of pediatric patients are required to evaluate the usefullness of Gharbi and WHO-IWGE Classification Systems for the choice of treatment.

\section{REFERENCES}

1. Moro P, Schantz PM. Echinococcosis: a review. Int J Infect Dis 2009; 13: 125-133.

2. Moro PL, Schantz PM. Echinococcus Species (Agents of Cystic, Alveolar, and Polycystic Echinococcosis). In: Long SS, Pickering LK, Prober CG (eds). Principles and Practice of Pediatric Infectious Diseases (4th ed). Philedephia: Elsevier, 2012: 1356-1362.

3. WHO Informal Working Group. International classification of ultrasound images in cystic echinococcosis for application in clinical and field epidemiological settings. Acta Trop 2003; 85: 253 261.

4. Djuricic SM, Grebeldinger S, Kafka DI, Djan I, Vukadin M, Vasiljevic ZV. Cystic echinococcosis in children - the seventeen-year experience of two large medical centers in Serbia. Parasitol Int 2010; 59: 257261.
5. Snábel V, Altintas N, D'Amelio S, et al. Cystic echinococcosis in Turkey: genetic variability and first record of the pig strain (G7) in the country. Parasitol Res 2009; 105: 145-154.

6. Yazar S, Yaman O, Cetinkaya F, Sahin I. Cystic echinococcosis in Central Anatolia, Turkey. Saudi Med J 2006; 27: 205-209.

7. Akalin S, Kutlu SS, Caylak SD, Onal O, Kaya S, Bozkurt AI. Seroprevalence of human cystic echinococcosis and risk factors in animal breeders in rural communities in Denizli, Turkey. J Infect Dev Ctries 2014; 8: 1188-1194.

8. Ok UZ, Ozkol M, Kilimcioğlu AA, et al. A provincebased study using sampling method to investigate the prevalence of cystic echinococcosis among primary school children in Manisa, Turkey. Acta Trop 2007; 103: 116-122.

9. Pakala T, Molina M, Wu GY. Hepatic echinococcal cysts: A review. J Clin Transl Hepatol 2016; 4: 39-46.

10. Calma CL, Neghina AM, Vlaicu B, Neghina R. Cystic echinococcosis in the human population of a western Romanian county, 2004-2010. Clin Microbiol Infect 2011; 17: 1731-1734.

11. Tenguria RK, Naik MI. Evaluation of human cystic echinococcosis before and after surgery and chemotherapy by demonstration of antibodies in serum. Ann Parasitol 2014; 60: 297-303.

12. Jordanova DP, Harizanov RN, Kaftandjiev IT, Rainova IG, Kantardjiev TV. Cystic echinococcosis in Bulgaria 1996-2013, with emphasis on childhood infections. Eur J Clin Microbiol Infect Dis 2015; 34: 1423-1428.

13. Çevik M, Eser I, Boleken ME. Characteristics and outcomes of liver and lung hydatid disease in children. Trop Doct 2013; 43: 93-95.

14. Bartels C, Beaute J, Fraser G, et al; European Centre for Disease Prevention and Control (ECDC) Annual epidemiological report 2014: food- and waterborne diseases and zoonoses. Stockholm: ECDC; 2014.

15. Schantz PM. Progress in diagnosis, treatment and elimination of echinococcosis and cysticercosis. Parasitol Int 2006; 55(Suppl): S7-S13.

16. Dopchiz MC, Elissondo MC, Andresiuk MV, et al. Pediatric hydatidosis in the south-east of the Buenos Aires province, Argentina. Rev Argent Microbiol 2009; 41: 105-111.

17. Agudelo Higuita NI, Brunetti E, McCloskey C. Cystic echinococcosis. J Clin Microbiol 2016; 54: 518-523.

18. Torgerson PR, Deplazes P. Echinococcosis: diagnosis and diagnostic interpretation in population studies. Trends Parasitol 2009; 25: 164-170. 
19. Wuestenberg J, Gruener B, Oeztuerk S, et al. Diagnostics in cystic echinococcosis: serology versus ultrasonography. Turk J Gastroenterol 2014; 25: 398404.

20. Tamarozzi F, Vuitton L, Brunetti E, Vuitton DA, Koch S. Non-surgical and non-chemical attempts to treat echinococcosis: do they work? Parasite 2014; 21: 75.
21. Brunetti E, Kern P, Vuitton DA; Writing Panel for the WHO-IWGE. Expert consensus for the diagnosis and treatment of cystic and alveolar echinococcosis in humans. Acta Trop 2010; 114: 1-16. 